\title{
Pile-up on X-ray CCD instruments
}

\author{
J. Ballet \\ DSM/DAPNIA/SAp, Bât. 709, CEA-Saclay, F-91191 Gif-sur-Yvette, France \\ e-mail: jballet@Cea.fr
}

Received February 12; accepted December 4, 1998

\begin{abstract}
This paper presents a statistical and analytical analysis of the pile-up phenomenon on X-ray CCD detectors, whereby two incoming X-rays are counted as one. The probability of measuring configurations involving 1 , 2,3 or 4 pixels is written down for a uniform incoming flux, thus allowing the computation of the flux loss (fraction of photons rejected because of pile-up) and of the pile-up fraction (fraction of events with wrong energy).

For detectors with pixels which are small compared to the point spread function (PSF) of the telescope (such as $X M M / E P I C-M O S$ or $A S C A / S I S)$ the formulae can be readily integrated over space to predict and account for pile-up on the total flux of point sources. It is shown that if only single events are selected the total pile-up fraction can never get very high for usual PSF shapes. The main effect is a loss of efficiency which is perfectly quantifiable. It is concluded that taking extreme care to avoid pile-up by adapting the instrument setting (for example restricting the useful area of the CCD) may not be so important after all, although it remains necessary if it is important to collect as many photons as possible from the source.

An extension to the theory is proposed for detectors with pixels comparable to the PSF width (such as $A X A F / A C I S)$, and for extended sources.
\end{abstract}

Key words: methods: analytical — methods: statistical — $\mathrm{X}$-rays: general

\section{Introduction}

After the $A S C A / S I S$, the next generation of X-ray astronomy satellites will all incorporate CCD detectors (AXAF/ACIS, SPECTRUM-X/JET-X, XMM/EPIC). The reason is that they are very versatile, offering good quantum efficiency, spatial resolution and reasonable energy resolution (Lumb et al. 1991).

The principle of an X-ray CCD detector is that the whole detector is read out at regular intervals. The contents of the detector as it is read out is called a frame.
A local excess in the charge contents is called an event, and is usually associated with a single X-ray impact. The total charge content of the event (in excess of the average) is directly proportional to the X-ray energy (with some dispersion of course), and is used as the energy measure.

The underlying hypothesis is that an event is due to only one X-ray. If an event is actually due to several superposed X-rays, then both the count rate and the energy measurements are wrong. This phenomenon (X-ray superposition) is known as pile-up. In order to avoid pileup, frames are read out as often as possible (typically every few seconds). Nevertheless, when the collecting area of the telescope is large and the point spread function (PSF) is good (desirable features for an X-ray satellite), there will always be sources bright enough that a large number of photons will concentrate in a small area of the CCD. For example, for the XMM/EPIC-MOS (Villa et al. 1996) the flux limit for using the instrument in normal (full CCD) mode with negligible pile-up is about 0.5 $10^{-2} \mathrm{cts} / \mathrm{pixel} /$ frame at maximum, or $0.15 \mathrm{mCrab}$ for a point source (Erd et al. 1996). This is a very severe limitation! There are ways to accelerate the CCD read-out, by reading only a selected area of the $\mathrm{CCD}$, but at the price of losing the area of sky corresponding to the rest of the CCD.

The aim of the current paper is to quantify the pile-up problem, and to investigate whether the effect is manageable, in the sense that it may still be possible to use data we know are affected by pile-up. In Sect. 2 and Appendix A: the probability of obtaining various pixel patterns (in $X M M$ parlance) or grades (in $A S C A$ parlance) is quantified for arbitrary input flux. The next sections apply these formulae to the case of point sources for detectors with pixels which are small compared to the PSF, such as $X M M / E P I C-M O S$ or $A S C A / S I S$. Section 3 and Appendix B: deal with the radial count rate profiles for a point source, and investigate the relationship between the integrated count rate and the input flux. Section 4 shows how to deal with a number of observational complications. Section 5 sums up the paper's results, as a guide 

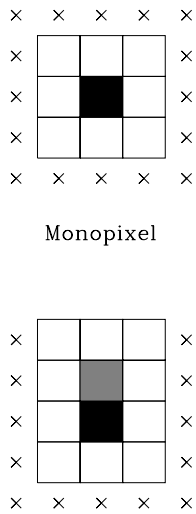

Bipixel
Monopixel

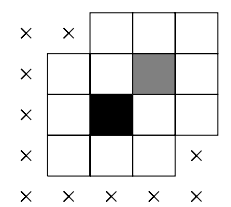

Diagonal

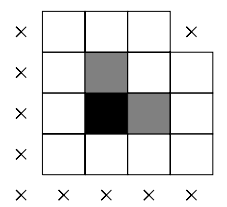

Tripixel

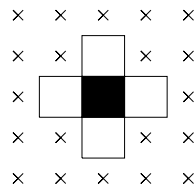

Mono +

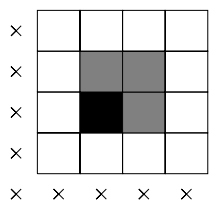

Quadripixel
Fig. 1. Possible charge patterns for X-ray events. The black pixel is the local maximum. The grey pixels are other pixels above threshold. The white pixels must be below threshold (they form the exclusion zone for secondary monopixels). The crosses denote pixels whose value does not influence the classification of the main event. The third pattern (called mono + ) is not necessarily isolated (it may include single events touching another one by a corner). The diagonal and mono + patterns are not produced directly by X-rays, but can occur as a consequence of pile-up

to observers. The numerical examples are given using parameters appropriate to the $X M M / E P I C-M O S$. Appendix $\mathrm{C}$ : compares the model with real data. Table 1 sums up all notations used in the paper.

\section{Patterns and local pile-up probability}

\subsection{General concepts}

The way to identify an event in a frame is to look for a local maximum above some statistical threshold, then to perform a proximity analysis around this maximum. The charge from an X-ray may either concentrate in a single pixel (monopixel events) or appear over several adjacent pixels (split events) forming a specific pattern or grade. The normal practise is to accept only isolated patterns (i.e. all pixels around the pattern, touching it even by a corner, must be below threshold).

True X-rays may appear in a finite number of charge patterns (Fig. 1):

1. Monopixels, with no other pixel above threshold around.

2. Bipixels, with two adjacent pixels above threshold.

3. Tripixels, with two pixels above threshold beside the maximum. They are necessarily L-shaped (aligned tripixels are not produced by true X-rays) and the corner pixel always receives the largest charge.

4. Quadripixels, forming a closed square (elongated fourpixel patterns are not produced by true X-rays).
Bipixels can be horizontal or vertical. Tripixels can have any of four orientations. One usually defines the position of a split event by that of the pixel with highest charge, allowing the definition of four orientations for bi- and quadripixels also. I call $\alpha_{i}$ the proportion of X-rays creating a charge pattern with $i$ pixels (this is also the pattern distribution of measured events in the ideal situation with no pile-up). The $\alpha_{i}$ usually depend on energy. The proportion of split events increases with energy for front illuminated CCDs such as XMM/EPIC-MOS. For back illuminated CCDs it is minimum at medium energy. Examples of $\alpha_{i}$ are $[0.778,0.195,0.014,0.013]$ at the energy of $\mathrm{Al}_{K}$ $(1.49 \mathrm{keV}),[0.459,0.303,0.046,0.192]$ at the energy of $\mathrm{Ni}_{\mathrm{K} \alpha}(7.48 \mathrm{keV})$, measured with the XMM/EPIC-MOS (EPIC MOS team, 1994). The situation where a sizable fraction of events is split over more than four pixels is discussed in Sect. 4.6.

For a relatively large incoming flux, two X-rays may materialize as monopixels in adjacent pixels and be counted as an apparent split event. This comes in addition to the true pile-up (two X-rays in the same pixel). In the following I compute the apparent pattern distribution (after pile-up), neglecting the possibility that small amounts of charge (below threshold) in peripheral pixels of two close-by charge patterns may combine to exceed threshold.

I call $\lambda$ the incoming X-ray flux/pixel/frame (assumed uniform and random) and $\mu_{j}$ the expected (expectation value of the) count rate/pixel/frame in pattern $j$ (to avoid ambiguities, I note $i$ charge patterns of incoming photons and $j$ patterns of detected events). Except in Appendix $\mathrm{C}$ the paper deals only with ideal (as opposed to noisy) quantities. The actual measured count rate would be $\mu_{j}$ with Poisson fluctuations.

I call $\mu_{j}^{\mathrm{t}}$ the expected count rate/pixel/frame in pattern $j$ of clean (not piled-up) events. $\mu_{j}^{\mathrm{t}}$ does not correspond to a measurable quantity, since one has no way to tell a clean event from a piled-up one a posteriori, but it is useful to know what it is. $1-\mu_{j} /\left(\alpha_{j} \lambda\right)$ is called the flux loss. It is the loss in detection efficiency due to pattern overlap. $1-\mu_{j}^{\mathrm{t}} / \mu_{j}$ is called the pile-up fraction. It is the fraction of measured events whose energy will be wrong. Note that in this paper I always refer to the flux loss and pile-up fraction per pattern. This is appropriate since the pattern is known in the data. Global quantities would be obtained by summing over the patterns.

In the next subsection and Appendix A: I derive the relationship between $\mu_{j}, \mu_{j}^{\mathrm{t}}, \lambda$ and $\alpha_{i}$. The formulae appear as a product of the probability to get such a pattern times the probability not to destroy it by receiving another photon in the exclusion zone around the "target" event. For $\mu_{j}^{\mathrm{t}}$, probability to get clean (not piled-up) events, the first (production) term is simply $\alpha_{j} \lambda$.

Since the probability not to receive a charge pattern of type $i$ in a pixel is always of the form $\exp \left(-\alpha_{i} \lambda\right)$, the second (suppression) term is always of the form $\exp \left(-\gamma_{j} \lambda\right)$, 


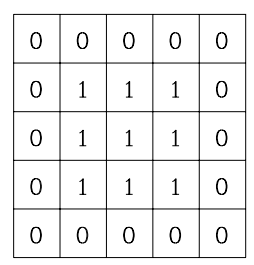

Monopixel

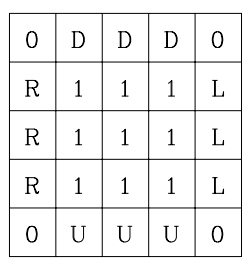

$\mathrm{Bi}-$ and tripixel

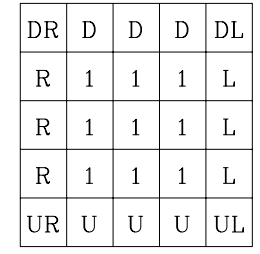

Quadripixel
Fig. 2. Exclusion zones for keeping a single event clean (not piled-up) as a function of the charge pattern of the secondary photon. R (L, U, D) stands for the fraction of patterns extending rightward (left, up, down) from the central pixel. For tri- and quadripixels the individual patterns are DR, DL, UR, UL which stand for the fraction of patterns extending down and rightward, etc. If the pattern distribution is symmetrical, $\mathrm{R}=\mathrm{L}=\mathrm{U}=\mathrm{D}=0.25$ for bipixels, 0.5 for tri- and quadripixels. $\mathrm{DR}=\mathrm{DL}=\mathrm{UR}=\mathrm{UL}=0.25$ for tri- and quadripixels. The total exclusion area (sum of all coefficients) is the same $(9,12,15,16$ for mono-, bi-, tri- and quadripixels) even if the distribution is not symmetrical

where $\gamma_{j}$ is a sum over all pixels in the exclusion zone (including the target event). I note $\gamma_{j}$ here the coefficient associated to $\mu_{j}^{\mathrm{t}}$ (clean events). For $\mu_{j}$ the exclusion zone would not include the target event, since additional charge patterns there would preserve the event's geometry. The exclusion zone is larger for larger patterns (because not one of their $i$ pixels must be close to the original pattern), so that $\gamma_{j}$ will appear as $\sum n_{j i} \alpha_{i}$, where $n_{j i}$ is the area of the exclusion zone for incoming pattern $i$ and measured pattern $j$. Mathematically, one may start by convolving the area which must be below threshold around the measured pattern $j$, plus the pattern itself (black, grey and white pixels in Fig. 1), with the area of the incoming pattern $i$ for a given orientation (black and grey pixels in Fig. 1). The exclusion zone (Fig. 2) may then be obtained by setting all pixels above 0 in the convolution to 1 , multiplying by the fraction of patterns with that orientation, and summing over all orientations.

For $\mu_{j}$, the production term must include all possibilities of pile-up preserving the geometrical shape of the pattern, and the exclusion zone does not include the target event.

The formulae have been checked against simulations (Ferrando et al. 1996) for various pattern distributions and incoming flux. Except for $\mu_{3}$ which is known to be approximate anyway (A.3.) I have seen no evidence of discrepancy. Early comparison to calibration data is presented in Appendix C.

\subsection{Monopixels}

I first consider the simplest case of monopixel events. For such an event to remain clean (not piled-up) there must be no other pixel above threshold in the exclusion zone
(Fig. 1, top left), nor in the target pixel. Therefore there must be no other monopixel pattern in the 9 corresponding pixels, whereas bipixels must be avoided over a larger area: the same 9 pixels, plus 3 pixels outside (on the left, top, right or bottom depending on the bipixel's orientation) from which the bipixel would spill over into a pixel immediately next to the event. The same reasoning leads to 15 pixels to be avoided for tripixels and 16 for quadripixels. Fig. 2 shows graphically the exclusion zone for all orientations of the secondary charge patterns. Its area is the same for all orientations because the target (monopixel) is symmetrical.

To get the production term for $\mu_{1}$, one can first say that monopixels can appear only from one or more superposed monopixels. The probability to have zero monopixels in a given pixel is $\mathrm{e}^{-\alpha_{1} \lambda}$. Therefore the probability to have at least one is $1-\mathrm{e}^{-\alpha_{1} \lambda}$. Finally the target pixel must be removed from the exclusion area for monopixels.

Finally, using $\alpha_{1}+\alpha_{2}+\alpha_{3}+\alpha_{4}=1$, one obtains:

$$
\begin{aligned}
\gamma_{1} & =9+3 \alpha_{2}+6 \alpha_{3}+7 \alpha_{4} \\
\mu_{1} & =\left(1-\mathrm{e}^{-\alpha_{1} \lambda}\right) \mathrm{e}^{-\left(\gamma_{1}-\alpha_{1}\right) \lambda} \\
\mu_{1} & =\left(\mathrm{e}^{\alpha_{1} \lambda}-1\right) \mathrm{e}^{-\gamma_{1} \lambda} \\
\mu_{1}^{\mathrm{t}} & =\left(\alpha_{1} \lambda\right) \mathrm{e}^{-\gamma_{1} \lambda}
\end{aligned}
$$

The probabilities to get other patterns are detailed in Appendix A.

\subsection{Measured count rate versus input photon flux}

From Eq. (2) (and those in Appendix A:) it is easy to plot, for a given pattern, the expected count rate as a function of incoming flux. Figure 3 illustrates this for the ideal case of a pure monopixel distribution. The maximum count rate is achieved for an incoming flux of

$\lambda_{0}=\frac{\left|\ln \left(1-\alpha_{1} / \gamma_{1}\right)\right|}{\alpha_{1}}$

For a pure monopixel distribution $\alpha_{1}=1$ (so $\gamma_{1}=9$ ), $\lambda_{0}=0.118$ and the maximum count rate is $4.3310^{-2}$. This corresponds to the maximum of the radial curve (Fig. 4). At this value of $\lambda$ the pile-up fraction (dotted curve) is still no larger than $5.77 \%$.

Because the expected count rate for a flat spatial distribution is not strictly increasing as a function of incoming photon flux, there is an ambiguity in reconstructing the incoming photon flux. This ambiguity can be overcome by considering at the same time the fraction of diagonal events over truly isolated monopixels (dashed line in Fig. 3). This is also by itself ambiguous, but matching both is not. At this kind of count rate the statistics (even in a single frame) is enough to distinguish solutions unambiguously, except at very high incoming flux, which is not a very interesting case. 


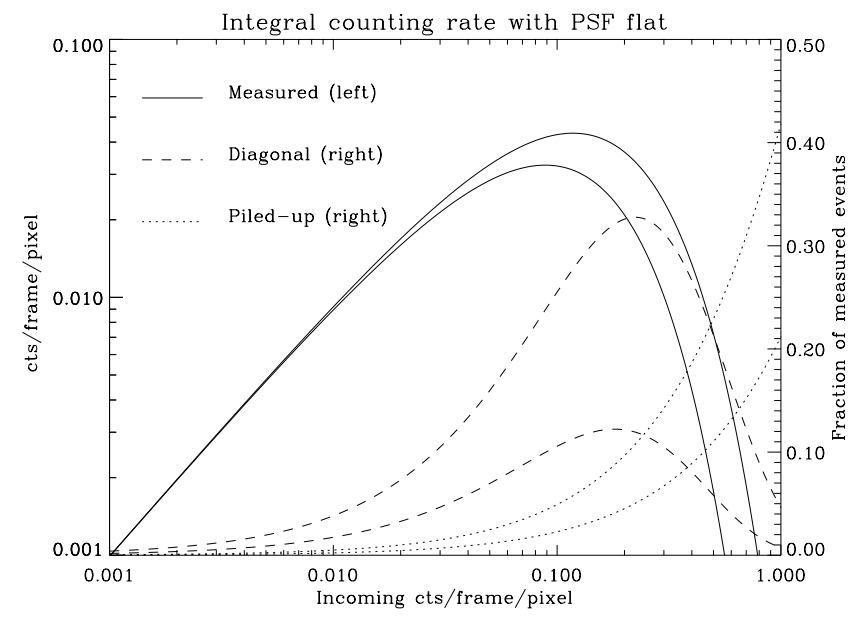

Fig. 3. Expectation value of the monopixel count rate (solid line, scale on the left axis) as a function of input photon flux for a flat spatial distribution. The dashed line is the ratio of single events in diagonal events to truly isolated monopixels (right axis). The dotted line is the fraction of piled-up single events (right axis). Two pattern distributions are superposed. The upper lines correspond to the ideal case of all monopixels. The lower lines correspond to the pattern distribution $[0.459$, $0.303,0.046,0.192]$ which includes larger events. For this case the monopixel count rate (solid line) was divided by $\alpha_{1}=0.459$ to allow easier comparison with the case of all monopixels. It is apparent that the presence of large events further suppresses the count rate, but reduces the proportion of diagonal events and of piled-up events

\section{Pile-up for point sources}

\subsection{Radial dependence}

Section 2 and Appendix A: fully describe the statistical properties of pile-up for a spatially uniform incoming flux. This situation can happen in the course of calibrations for instance, or for large extended sources. However the most important case for astronomical observations is that in which point sources are projected onto the detector, spread by the PSF of the telescope.

If the half width at half maximum (HWHM) of the PSF is safely larger than the pixel size (as for $X M M / E P I C-M O S$ for instance), then it is possible to use Eqs. (2-3) locally, and estimate pile-up at any point in the PSF. Note that the formulae for monopixels, which are the most important as is shown next, depend on the incoming $\mathrm{X}$-ray flux at distance $\sqrt{2}$ pixel at most. For $X M M / E P I C$ $M O S$ it is expected that the HWHM will be about 4 pixels. Therefore for a source centred on a pixel, the flux (per pixel) in the eight neighbouring pixels will be about $10 \%$ lower than that in the central pixel, so that formulae (1-3) will overestimate pile-up there. But the central pixel receives no more than $1 \%$ of the total source flux, and for other pixels the problem is much less severe. The opposite case of pixels comparable to the HWHM of the PSF is discussed in Sect. 4.4.

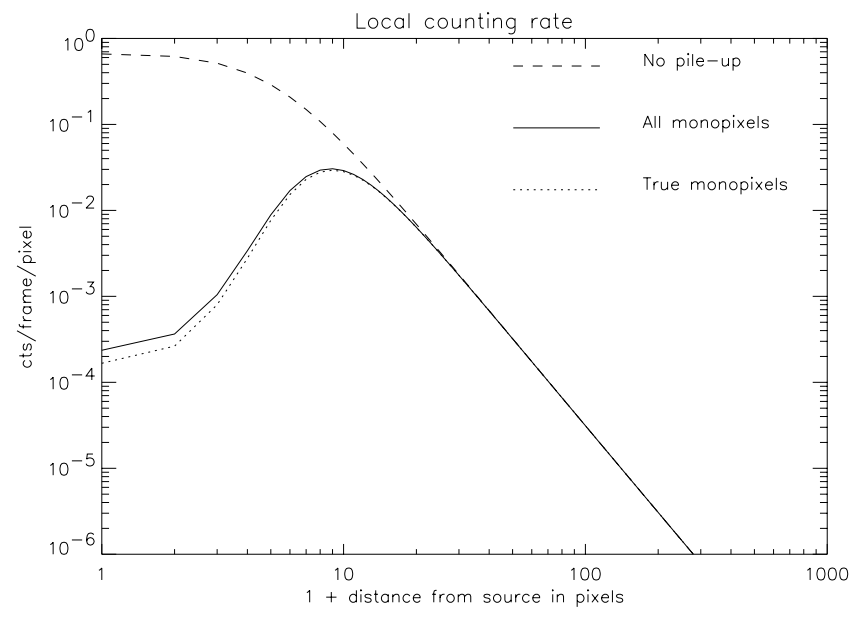

Fig. 4. Expected radial distribution of the monopixel count rate (solid line), as opposed to the distribution one would get in the absence of pile-up (dashed line) and that of clean (not piled-up) monopixels (dotted line). The point spread function (PSF) follows a King profile $(g(r)$ defined in $(\mathrm{B} 1))$ with $\beta=$ $10 / 3$ and $r_{0}=5$ (diameter at half maximum $=7.2$, at half encircled energy $=13.5$ pixels). The total input source flux is 100 photons/frame. The pattern distribution is [0.778, 0.195, $0.014,0.013]$. The global flux loss is $61.42 \%$. The global pile-up rate is $1.63 \%$

Figure 4 shows an example of the expected count rate for an axisymmetric PSF with radial profile representative of the $X M M / E P I C-M O S$, a low-energy pattern distribution (the relevant pattern distribution is that averaged over energy, which is dominated by the more numerous low-energy photons) and a large source flux. What is important to note is that even though the flux loss is large $(61.42 \%)$ as expected for such a bright source, the pileup fraction remains very modest at $1.63 \%$. This is because the probability of a true monopixel pile-up is much less (only 1 pixel target area) than that of destroying the monopixel (8 pixels target area for other monopixels, and more for bi-, tri- and quadripixels). Adding the diagonal monopixels (Eqs. A3-A4) reduces the flux loss to $58.56 \%$, but increases the pile-up fraction to $2.02 \%$. Counting as good events monopixels touching some other pattern by a corner (Eq. (A5)) reduces further the flux loss to $51.80 \%$, while increasing the pile-up fraction to $2.76 \%$. Note that the figures given here are for integration to 1000 pixels distance, but they do not change a lot if it is performed to 50 pixels $\left(10 r_{0}\right)$ only ( $4.5 \%$ of the flux falls beyond 50 pixels).

Figure 5 shows the same plot for bipixels. In this case the flux loss is comparable (57\%), but the pile-up fraction is much higher $(21 \%)$. This is because for this (typical) pattern distribution there are four times more monopixels than bipixels, thus leading to a large fraction of adjacent monopixels being counted as bipixels. The figures are somewhat similar for tripixels and quadripixels. 


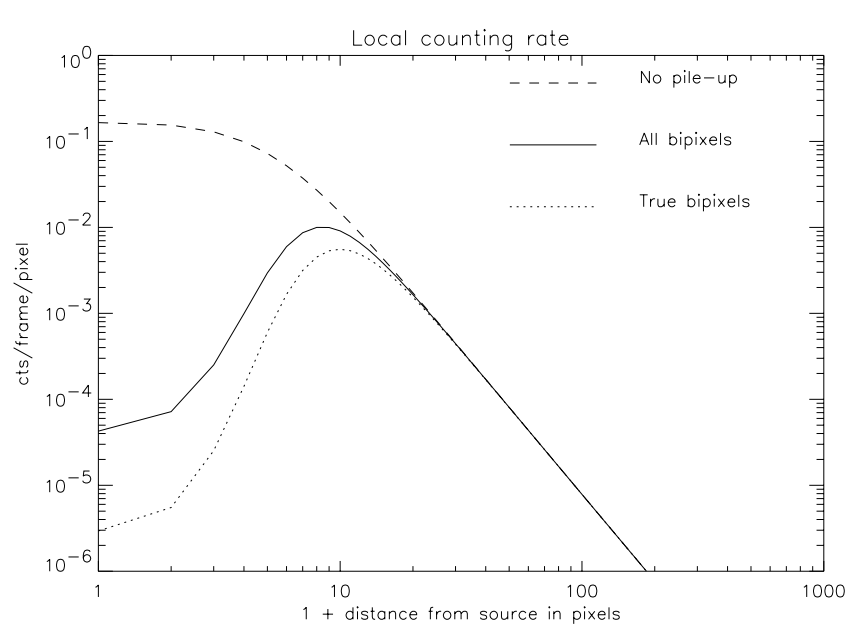

Fig. 5. Same as Fig. 4 for bipixel events, using formulae (A6-A7). The global flux loss is $57.15 \%$. The global pile-up rate is $21.30 \%$, much larger than for monopixels because the clean bipixel events are dominated near the center by adjacent monopixel events counted as one bipixel event

For a less extreme source flux, such as 10 photons/frame, the flux loss and pile-up rate for monopixels are $18.40 \%$ and $0.74 \%$, respectively. Including diagonal events, the figures are $14.45 \%$ and $0.86 \%$. For bipixels the flux loss is $12.65 \%$ and the pile-up rate $12.43 \%$.

The main conclusion is that flux loss and transfer of single events to bipixels or larger is the dominant effect of pile-up. For all spectral applications when pile-up is suspected to have occurred it is much safer to select only monopixel events. Diagonal events may be used, but never total up a large fraction of the true monopixels. However, rejecting all split events incurs a severe loss of effective area at high energy (a factor of 2), where the number of events may not be large even though the number of low-energy photons exceeds the pile-up limit.

\subsection{Measured count rate versus source flux}

After integrating the local rates from equations (2-3) over space (any spatial domain is allowed, but here I consider the full space), one obtains the count rate of clean (not piled-up) monopixel events $M_{1}^{\mathrm{t}}$ and the full expected monopixel count rate $M_{1}$ as a function of the incoming source flux. I call the integral source flux (/frame) $\Lambda$, to avoid confusion with the local flux (/pixel/frame) $\lambda$.

$$
\begin{aligned}
& M_{1}^{\mathrm{t}}=\iint \alpha_{1} \Lambda g(x, y) \mathrm{e}^{-\gamma_{1} \Lambda g(x, y)} \mathrm{d} x \mathrm{~d} y \\
& M_{1}=\iint\left(\mathrm{e}^{\alpha_{1} \Lambda g(x, y)}-1\right) \mathrm{e}^{-\gamma_{1} \Lambda g(x, y)} \mathrm{d} x \mathrm{~d} y .
\end{aligned}
$$

The result is shown in Fig. 6 for the same PSF $g(x, y)$ as used in Fig. 4. It is immediately apparent that the total count rate never decreases (contrary to the flat case), because the wings always more than compensate for the

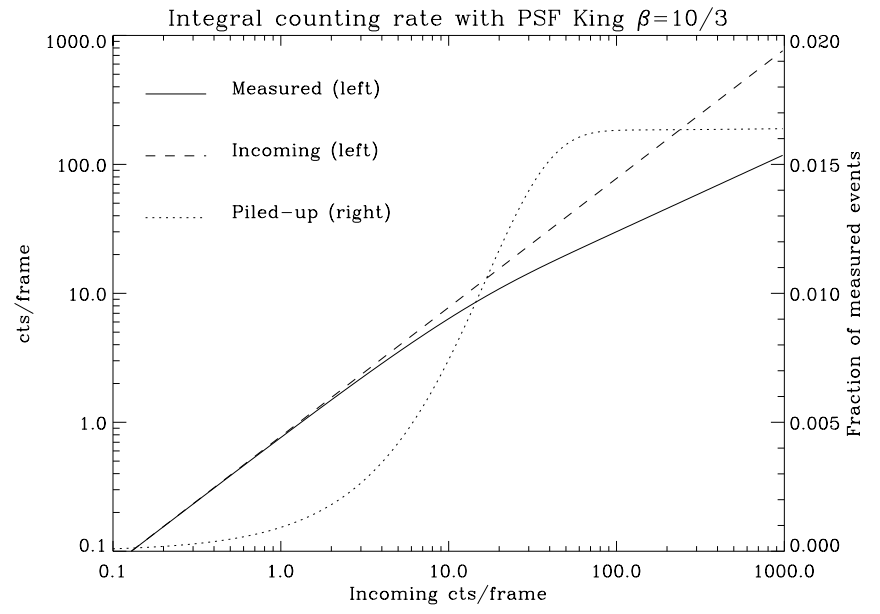

Fig. 6. Expectation value of the monopixel count rate (integrated over the detector, solid line) as a function of total input source flux for a PSF following a King profile with $\beta=10 / 3$ and $r_{0}=5$. The pattern distribution is $[0.778,0.195$, $0.014,0.013]$. The scale is on the left axis. The dashed line shows the input flux multiplied by $\alpha_{1}$ (no pile-up) for comparison. The dotted line is the fraction of piled-up events (right axis). This figure shows how the pile-up fraction tends to a constant at large source flux. The fraction of diagonal events (not shown) also tends to a constant at high flux, $7.5 \%$ in this case

flux loss in the center. The pile-up fraction (dotted line) increases then saturates at a constant value. Appendix B: explains analytically why it is so, and offers a similar analysis for a Gaussian PSF.

This has two important consequences. First, because the expected count rate is a strictly increasing function of the input source flux it is possible to estimate quite precisely the source flux from the measured count rate, and model the piled-up PSF, similarly to "curve of growth" analysis for absorption lines. Secondly, because the pileup fraction among monopixel events remains small, a spectrum restricted to monopixels is not hopelessly corrupted, even for very bright sources. The detailed study of the spectral perturbations induced by pile-up is deferred to a later paper.

It is also useful to know how pile-up behaves at low flux, allowing one to estimate when it can be safely ignored. Developing Eqs. (6) and (5) over $\Lambda$ to order 2, one easily obtains for the flux loss and the pile-up rate on single events:

$$
\begin{aligned}
& 1-\frac{M_{1}}{\alpha_{1} \Lambda}=\Lambda\left(\gamma_{1}-\frac{\alpha_{1}}{2}\right) \iint g(x, y)^{2} \mathrm{~d} x \mathrm{~d} y \\
& 1-\frac{M_{1}^{\mathrm{t}}}{M_{1}}=\Lambda \frac{\alpha_{1}}{2} \iint g(x, y)^{2} \mathrm{~d} x \mathrm{~d} y .
\end{aligned}
$$

This shows first that pile-up is a linear phenomenon at low flux, so that there is no obvious threshold below which it is negligible. The ratio between flux loss and pile-up rate $\left(2 \gamma_{1} / \alpha_{1}-1\right)$ does not depend on the PSF and is at least 17 (for $\alpha_{1}=1$, all other $\alpha_{i}=0$ ), showing once 
again that flux loss is much more important than genuine pile-up. The relationship between source flux (/frame), $\Lambda$, and flux loss depends on the PSF. For the same King PSF and pattern distribution as in Fig. 6 (appropriate for $X M M / E P I C-M O S$ ), the coefficient relating flux loss to $\Lambda$ is about $2.210^{-2}$. If one sets a threshold at $1 \%$ flux loss, the count rate must not be larger than $0.5 \mathrm{cts} /$ frame, or $410^{-3} \mathrm{cts} / \mathrm{pixel} /$ frame at maximum, indeed very similar to the estimate in Erd et al. (1996).

\section{Complications}

\subsection{X-ray background}

Section 3 on point sources implicitly neglects the (more or less uniform) X-ray background which is superposed on the source. This is justified because pile-up most strongly affects bright sources, well above the background. Quantitatively, for $X M M / E P I C$ - $M O S$ the background rate is expected to be $\lambda_{0} \simeq 10^{-6} \mathrm{cts} / \mathrm{pixel} /$ frame, to be compared to the threshold for pile-up estimated in Sect. 3.2 to be $410^{-3} \mathrm{cts} / \mathrm{pixel} /$ frame at maximum. For the King PSF appropriate to XMM/EPIC-MOS, the local count rate for a source at threshold crosses the background level at $r_{\text {eq }} \simeq 50$ pixels distance. Within this circle, the background totals about $10^{-2} \mathrm{cts} /$ frame, and the source about 0.5 cts/frame.

To take this into account properly, one would have to replace $\Lambda g(x, y)$ with $\Lambda g(x, y)+\lambda_{0}$ throughout and stop the integration outwards somewhere around $r_{\text {eq }}$.

\subsection{Finite spectral range}

The pile-up process is a purely probabilistic effect which does not depend on the energy of the photons, except through the pattern distribution. But it affects the energy measurement which is the sum of the energies of all photons which have contributed to the measured event. Section 3 implicitly assumes that the energy band of the instrument is unbounded to high energies. However $\mathrm{X}$-ray instruments usually incorporate some kind of high energy threshold above which events are discarded, so that piled-up events with large total charge are not counted. Therefore the measured count rate is actually lower than estimated in Eq. (6). Quantitatively, this is always a very small effect. A simulation for XMM/EPIC-MOS with a relatively hard spectrum $\left(N_{\mathrm{H}}=10^{21} \mathrm{~cm}^{-2}\right.$, photon index 1.5 ) shows that only a few $\%$ of piled-up events get above the threshold. Since for monopixels the proportion of piled-up events is itself around 1\%, the effect is on the order of a few $10^{-4}$.

\subsection{Attitude drifts}

It often happens that the telescope's attitude is not perfectly stable. It oscillates around the average pointing direction. What is important for the pile-up phenomenon (and for the spatial resolution) is what happens within a single frame. Therefore any attitude drift on time scales large compared to the frame integration time is irrelevant. The events may be accumulated in sky coordinates (i.e. corrected for the drifts) and pile-up analysed as if there were no drifts.

On the other hand, if non-negligible drifts occur during the frame integration time, then the PSF used for analysing the image (including the pile-up phenomenon) must be broadened to account for that. The bottom line is that pile-up depends on the effective PSF.

\subsection{Pixels comparable to the PSF width}

When the half width at half maximum (HWHM) of the PSF is not much larger than the pixel size (as for $A X A F / A C I S$ or $X M M / E P I C-P N)$, Eq. (6) cannot be applied directly because the probability for a secondary X-ray to fall in the pixel just next to the peak of the PSF can be much lower than exactly on the peak, thereby violating the assumptions used in Sect. 2 for deriving the suppression factor.

However the reasoning of Sect. 2 can be generalized to non-uniform fluxes. For monopixels at least, only the suppression term is affected (the production term involves only one pixel). $\lambda$ should be replaced by $\Lambda g(x, y)$ (where $g(x, y)$ is the normalized PSF $)$. Then instead of $\gamma_{1} \lambda$ in (2-3) one gets $\sum \alpha_{i} \Lambda g\left(x^{\prime}, y^{\prime}\right)$ where the sum bears on all patterns and for each pattern on all pixels of the exclusion zone. This is actually the convolution of $g(x, y)$ with the exclusion masks $Z_{1 i}(x, y)$ of Fig. 2 (the mask's integrals are the $n_{j i}$ coefficients of Sect. 2.1). Therefore

$$
\begin{aligned}
& Z_{1}(x, y)=\sum_{i} \alpha_{i} Z_{1 i}(x, y) \\
& \mu_{1}(x, y)=\left(\mathrm{e}^{\alpha_{1} \Lambda g(x, y)}-1\right) \mathrm{e}^{-\Lambda\left(Z_{1} * g\right)(x, y)}
\end{aligned}
$$

where $Z_{1}$ is the effective exclusion mask for monopixel targets. It is of course important to use the PSF projected on pixels for the exact source position (which can be at the centre of a pixel, or on a side).

This leads in general to less flux loss at the center of the PSF but more pile-up than predicted by (2-3). For example, integrating (9) over space for a King PSF with $r_{0}=0.6$ pixels and $\beta=10 / 3$ (representative of the $A X A F / A C I S$ ), and $\Lambda=1$ count/frame, leads to a flux loss of $37.8 \%$ for monopixels and a pile-up rate of $4.6 \%$ when the source is centered on a pixel (the least favourable case). Equations (5) and (6) would have predicted a flux loss of $52.5 \%$ and a pile-up rate of $1.6 \%$. For that PSF the 
pile-up rate reaches a maximum of $8 \%$ or so at $\Lambda \simeq 3$ photons/frame, after which it declines to get back to values predicted by the simple model (5-6).

For a PSF representative of the $X M M / E P I C-M O S$, with $\Lambda=10$ photons/frame, the error incurred by using (2) instead of (9) is about $7 \%$ in the central pixel, and about $0.5 \%$ when integrated over the whole spatial domain as in (6). This justifies using the simpler formulae in that case ( $\mathrm{HWHM}=3.6$ pixels).

\subsection{Extended sources}

If the shape of the source $R(x, y)$ is known in advance and the spectrum is constant over the source, then the problem is similar to that of a point source, simply replacing the PSF $g(x, y)$ by the convolution $R * g$ in Eq. (6), which applied over the whole spatial domain allows to estimate precisely the true source flux (/frame), $\Lambda$, from the measured count rate.

In the general case, Eq. (2) may still be applied locally (at the pixel or few-pixel scale) to try and recover the true spatial distribution. However one is then hampered by the statistics, and care must be taken to estimate the errors properly in the heavily piled-up areas (the pile-up correction destroys the Poisson character). This problem is actually very similar to that of retrieving the PSF from real data, presented in Appendix C.2.

\subsection{Special pattern distributions}

The formulae of Appendix A assume that the pattern distribution is symmetrical (i.e. as many vertical bipixels as horizontal ones, an equal number of tripixels in all four orientations). If this is not true then each orientation must be considered independently, making the formulae longer, although the principle remains the same. This has no influence on monopixels, for which only the total number of bi-, tri- and quadripixels is important. Therefore the simple formulae of Sect. 3 are not affected.

In some situations it may happen that true X-rays split over more than four pixels. For example this is the case for $X M M / E P I C-M O S$ at high energy when an X-ray is absorbed in the field free region of the CCD. The charge is then collected over many pixels (up to several tens). This is not usually a severe problem because even though it affects a large (up to one half) fraction of high energy photons, pile-up depends on the overall pattern distribution (integrated over the source spectrum) which is dominated by low-energy photons.

However this can be taken into account perfectly. These large patterns affect only the suppression terms (such events are not collected, they are of poor quality). It is thus enough to compute the exclusion areas for large patterns, to be added to $\gamma_{j}$ in (1) or Appendix A. On the other hand it would be extremely tedious to include in the formula coefficients for all possible shapes and sizes, so reasonable approximations are in order. As an example, if these charge patterns appear compact (i.e. of minimal linear extension for a given area), the exclusion areas for monopixel targets and secondary patterns with $N=5$, $6,7,8,9$ pixels are respectively $A=19,20,23,24,25$ pixels. An approximate formula, valid to \pm 1 pixel up to $N=1000$, is

$A=N+4 \sqrt{N}+5$.

\section{Summary}

This section is restricted, as Sect. 3, to instruments with pixel size small compared to the PSF.

\subsection{Planning the observation}

If one knows that the target will be piled-up because it exceeds the threshold of Sect. 3.2, the count rate must be maximized (because one is interested in time variations, or in weak spectral features), and there is nothing of interest in the rest of the CCD, then it is better to select a window on the CCD to reduce the frame integration time (directly linked to the window size) down to the level when pile-up is not a problem, and/or to use a spectral filter to cut-off low-energy photons. It is also advised to take strong steps to avoid pile-up if the source is extended and its shape not known.

If, on the other hand, the source is not expected to be bright, then it is not worth reducing the CCD window simply as a precaution. The above methods will allow use of the data at times when the source is bright. It can also be applied if the count rate is too high even in window mode and the guest observer does not want to lose spatial information (as would be the case by using timing mode).

It is also not worth taking special precautions to avoid pile-up if the bright source is not the target, except if it is so close that the target sits on the wings of the bright source.

\subsection{Analysing the data}

The first thing to do is to check for the occurrence of pile-up in the data, by systematically checking the local count rate (/frame) of monopixel events (averaged over PSF sized areas, to reduce statistical fluctuations) against the threshold defined in Sect. 3.2, equating the flux loss of Eq. (7) to $1 \%$ or, more generally, to the precision required. The average over time must be performed in sky coordinates. If the threshold is exceeded then pile-up must be taken into account. 
The second thing to do is to estimate the pattern distribution. One should not use the observed distribution (corrupted by pile-up), but take the energy spectrum $S_{1}(E)$ of single events only, then deduce the pattern distribution in the data $\overline{\alpha_{i}}$ from that $\alpha_{i}(E)$ calibrated as a function of energy, according to

$\overline{\alpha_{i}}=\frac{\int_{E} S_{1}(E) / \alpha_{1}(E) \alpha_{i}(E) \mathrm{d} E}{\int_{E} S_{1}(E) / \alpha_{1}(E) \mathrm{d} E}$.

The third thing to do is to check whether the source is point-like for the instrument. This may be obvious if it is known to be a compact source. If nothing is known, then from Eq. (6) (Fig. 6) it is possible to estimate the incoming flux from the measured count rate in the hypothesis of a point source $\left(\gamma_{1}\right.$ being deduced from the $\overline{\alpha_{i}}$ as in (1)). Comparing then the observed radial profile with the theoretical one for a source at that flux - the integrand of Eq. (6) - it is possible to check the point source hypothesis. If the source is extended then see Sect. 4.5.

Equation (6) may be used over any spatial domain, and can be generalized to split events using the formulae in Appendix A. However it can be used reliably only over the full spectral domain. All detected charge patterns contribute to pile-up, whatever their energy. In particular any spectral selection on events done after the detection process (in the course of the data analysis or even on-board) will not erase its effects. Once the overall input source flux (/frame) is known, and the spectrum is estimated from $S_{1}(E) / \alpha_{1}(E)$, a simulation may be carried out to quantify the spectral effects of pile-up. An analytical estimate is also possible. This will be the subject of a future paper.

Acknowledgements. It is a pleasure to acknowledge numerous discussions about pile-up with J.L. Sauvageot and P. Ferrando. I am also indebted to J. Osborne and to the referees M. Popp and L. Strueder, who suggested many ways to make the paper clearer.

\section{Appendix A: Pile-up probability for split events}

To avoid lengthy equations I denote as $p_{1}$ and $p_{2}$ the probability to produce a monopixel event (from one or more monopixels) and a vertical (or horizontal) bipixel event (from one or more vertical or horizontal bipixels).

$p_{1}=1-\mathrm{e}^{-\alpha_{1} \lambda}$
$p_{2}=1-\mathrm{e}^{-\alpha_{2} \lambda / 2}$.

\section{A.1. Diagonal and extended monopixels}

It is interesting to derive the rate of diagonally attached monopixels (two monopixels touching by a corner and globally isolated. See Fig. 1, top centre). Those are produced only by piled-up monopixels (and $\mathrm{Si}_{\mathrm{K}}$ escape, which can be recognized because one of the energies is that of $\mathrm{Si}_{\mathrm{K}}$ ). Therefore they can reliably be counted as two
Table 1. Summary of notations used in the paper. Symbols used only locally are not necessarily included

\begin{tabular}{|c|c|c|}
\hline Symbol & Meaning of the symbol & See \\
\hline$\overline{\overline{\alpha_{i}}}$ & average of $\alpha_{i}$ over energy & $(10)$ \\
\hline$\alpha_{i}$ & fraction of charge patterns with $i$ pixels & 2.1 \\
\hline$\beta$ & power law coefficient for King profile PSF & (B1) \\
\hline$r_{0}$ & core radius (sigma) for King (Gauss) PSF & (B1) \\
\hline$g(x, y)$ & $\begin{array}{l}\text { spatial distribution of incoming flux } \\
\text { (PSF) }\end{array}$ & 3.2 \\
\hline$\gamma_{1}$ & $\begin{array}{l}\text { suppression coefficient for clean single } \\
\text { events }\end{array}$ & $(1)$ \\
\hline$\gamma_{j}$ & $\begin{array}{l}\text { suppression coefficient for clean events } \\
\text { with pattern } j\end{array}$ & 2.1 \\
\hline$\Gamma$ & Gamma function & $(\mathrm{B} 2)$ \\
\hline$i$ & charge pattern of incoming photon & 2.1 \\
\hline$j$ & pattern of detected event & 2.1 \\
\hline$\lambda$ & incoming X-ray flux/pixel/frame & 2.1 \\
\hline$\lambda_{0}$ & $\begin{array}{l}\text { photon flux (/pixel/frame) for maximum } \\
\text { monopixel count rate }\end{array}$ & $(4)$ \\
\hline$\Lambda$ & integral source flux/frame & 3.2 \\
\hline$\mu_{1}$ & monopixel count rate after pile-up & $(2)$ \\
\hline$\mu_{j}$ & count rate after pile-up in pattern $j$ & 2.1 \\
\hline$\mu_{1}^{\mathrm{t}}$ & clean (not piled-up) monopixel count rate & $(3)$ \\
\hline$\mu_{j}^{\mathrm{t}}$ & $\begin{array}{l}\text { clean (not piled-up) count rate in pattern } \\
j\end{array}$ & 2.1 \\
\hline$M_{1}$ & $\begin{array}{l}\text { integral count rate after pile-up in pat- } \\
\text { tern } j\end{array}$ & $(6)$ \\
\hline$M_{1}^{\mathrm{t}}$ & $\begin{array}{l}\text { clean (not piled-up) integral monopixel } \\
\text { count rate }\end{array}$ & $(5)$ \\
\hline$n_{j i}$ & $\begin{array}{l}\text { area of the exclusion zone for incoming } \\
\text { pattern } i \text { and measured pattern } j\end{array}$ & 2.1 \\
\hline$p_{1}$ & $\begin{array}{l}\text { probability to produce a monopixel event } \\
\text { (no suppression) }\end{array}$ & $(\mathrm{A} 1)$ \\
\hline$p_{2}$ & $\begin{array}{l}\text { probability to produce a vertical (or hor- } \\
\text { izontal) bipixel event (no suppression) }\end{array}$ & $(\mathrm{A} 1)$ \\
\hline$r$ & radius from source centre & $\mathrm{B} 1$ \\
\hline$R(x, y)$ & intrinsic source spatial shape & 4.5 \\
\hline$S_{1}(E)$ & $\begin{array}{l}\text { source energy spectrum }(\mathrm{cts} / \mathrm{keV}) \text { re- } \\
\text { stricted to single events }\end{array}$ & 5.2 \\
\hline$Z_{1}$ & $\begin{array}{l}\text { effective exclusion zone for monopixel tar- } \\
\text { gets }\end{array}$ & $(9)$ \\
\hline$Z_{1 i}$ & $\begin{array}{l}\text { exclusion zone for incoming pattern } i \text { and } \\
\text { monopixel targets }\end{array}$ & 4.4 \\
\hline
\end{tabular}

monopixels. The exclusion area corresponding to clean (not piled-up) diagonal events, derived in the same way as in 2.2 , is 14 for monopixels, 18 for bipixels, 21 or 22 for tripixels (depending on their orientation), and 23 for quadripixels. The production probability is the square of that of monopixels, times 4 (there are 4 corners), both for $\mu_{1}^{\mathrm{d}}$ (total) and for $\mu_{1}^{\mathrm{dt}}$ (clean). Note that the rate of diagonal events (comprising two monopixels) is half that.

$$
\begin{aligned}
\gamma_{1}^{\mathrm{d}} & =14+4 \alpha_{2}+7.5 \alpha_{3}+9 \alpha_{4} \\
\mu_{1}^{\mathrm{d}} & =4 p_{1}^{2} \mathrm{e}^{-\left(\gamma_{1}^{\mathrm{d}}-2 \alpha_{1}\right) \lambda} \\
\mu_{1}^{\mathrm{d} \mathrm{t}} & =4\left(\alpha_{1} \lambda\right)^{2} \mathrm{e}^{-\gamma_{1}^{\mathrm{d} \lambda}} .
\end{aligned}
$$

For completeness I note that if one relaxes the constraint that a monopixel be fully isolated, so as to include 
monopixels touching anything else by a corner (Fig. 1, top right), only $\gamma_{1}$ is changed in Eqs. (2-3) to become

$\gamma_{1}^{\mathrm{c}}=5+3 \alpha_{2}+5 \alpha_{3}+7 \alpha_{4}$.

These events are less reliable, though, because they can also be produced by cosmic-rays.

\section{A.2. Bipixels}

For clean bipixels (Fig. 1, bottom left) the exclusion areas are $[12,15.5,19,20] .15 .5$ for bipixels is actually 15 for bipixels parallel to that considered and 16 for perpendicular bipixels. For $\mu_{2}$ (all bipixels) one must remove the two pixels of the pattern for monopixels, and one pixel for parallel bipixels, from the exclusion areas. The probability to produce a bipixel pattern of a given orientation (vertical, say), is that of having at least one vertical bipixel there $\left(p_{2}\right)$ plus in the remaining cases $\left(1-p_{2}\right)$ that of having at least two adjacent monopixels $\left(p_{1}^{2}\right)$.

$$
\begin{aligned}
\gamma_{2} & =12+3.5 \alpha_{2}+7 \alpha_{3}+8 \alpha_{4} \\
\mu_{2} & =2\left[p_{2}+\left(1-p_{2}\right) p_{1}^{2}\right] \mathrm{e}^{-\left(\gamma_{2}-2 \alpha_{1}-0.5 \alpha_{2}\right) \lambda} \\
\mu_{2}^{\mathrm{t}} & =\left(\alpha_{2} \lambda\right) \mathrm{e}^{-\gamma_{2} \lambda} .
\end{aligned}
$$

\section{A.3. Tripixels}

For clean tripixels (Fig. 1, bottom center) the exclusion areas are $[15,19,22.75,24] .22 .75$ is due to the fact that the exclusion area is not the same for tripixels having exactly reversed orientation as that considered (22) and all others (23). For $\mu_{3}$ (all tripixels) one must remove the three pixels of the pattern for monopixels, one pixel for bipixels of all orientations and one pixel for identical tripixels, from the exclusion areas.

For tripixels there is a complication since the easiest thing to compute is the probability $\mu_{3}^{\mathrm{g}}$ to get a geometrical tripixel, but what one is really interested in is the probability $\mu_{3}$ to get a tripixel with maximum charge at the corner (other tripixels cannot be confused with a regular X-ray and can be rejected straight away). $T_{3}^{\mathrm{g}}$ (and $T_{3}$ ) is the probability to construct a tripixel from monoand bipixels. It is written as the sum of that with no bipixel (requiring three monopixels), plus that with one (or more) bipixel of a single orientation (requiring one additional monopixel), plus that with bipixels of both orientations. I obtain an estimate of $T_{3}$ by noting that a) exactly one third of tripixels from three monopixels have maximum energy at the corner; b) for tripixels formed from one bipixel plus one monopixel only half the bipixels have their maximum charge at the corner, and that charge has at most a $50 \%$ chance of being larger than that of the monopixel; and c) for tripixels formed from two bipixels only the case when none of the bipixels has maximum charge at the corner is excluded. The last two contributions to $T_{3}$ (b and c) can only be estimated approximately.

$$
\begin{aligned}
\gamma_{3} & =15+4 \alpha_{2}+7.75 \alpha_{3}+9 \alpha_{4} \\
\gamma_{3}^{\prime} & =\gamma_{3}-3 \alpha_{1}-\alpha_{2}-0.25 \alpha_{3} \\
T_{3}^{\mathrm{g}} & =p_{1}^{3} \mathrm{e}^{-\alpha_{2} \lambda}+2 p_{1} p_{2} \mathrm{e}^{-\frac{\alpha_{2} \lambda}{2}}+p_{2}^{2} \\
\mu_{3}^{\mathrm{g}} & =4\left[1-\mathrm{e}^{-\frac{\alpha_{3} \lambda}{4}}\left(1-T_{3}^{\mathrm{g}}\right)\right] \mathrm{e}^{-\gamma_{3}^{\prime} \lambda} \\
T_{3} & \simeq \frac{p_{1}^{3}}{3} \mathrm{e}^{-\alpha_{2} \lambda}+\frac{p_{1} p_{2}}{2} \mathrm{e}^{-\frac{\alpha_{2} \lambda}{2}}+\frac{3 p_{2}^{2}}{4} \\
\mu_{3} & =4\left[1-\mathrm{e}^{-\frac{\alpha_{3} \lambda}{4}}\left(1-T_{3}\right)\right] \mathrm{e}^{-\gamma_{3}^{\prime} \lambda} \\
\mu_{3}^{\mathrm{t}} & =\left(\alpha_{3} \lambda\right) \mathrm{e}^{-\gamma_{3} \lambda} .
\end{aligned}
$$

\section{A.4. Quadripixels}

For clean quadripixels (Fig. 1, bottom right) the exclusion areas are $[16,20,24,25]$. For $\mu_{4}$ (all quadripixels) one must remove the four pixels of the pattern for monopixels, two pixels for bipixels of any orientation and one pixel for tri- and quadripixels, from the exclusion areas. $Q_{3}$ below is the probability to get a quadripixel event from mono-, bi- and tripixels. $Q_{2}$ is the probability to get a quadripixel event from mono- and bipixels only. In $Q_{3}$ the second term (subtracted) is the probability to have no tripixel and not the right combination of mono- or bipixels. The rightmost term (also subtracted) is the probability to have one (or more) tripixel of a single orientation and no bipixel or monopixel filling the hole. All other combinations form a quadripixel. $Q_{2}$ is written as the sum of the probability to get a quadripixel with no bipixel (requiring four monopixels), plus that with a bipixel at a single place in a single orientation (requiring two additional monopixels), plus that with bipixels of both orientations but no two parallel bipixels (requiring one additional monopixel), plus that with two parallel bipixels.

$$
\begin{aligned}
\gamma_{4} & =16+4 \alpha_{2}+8 \alpha_{3}+9 \alpha_{4} \\
Q_{2} & =p_{1}^{4} \mathrm{e}^{-2 \alpha_{2} \lambda}+4 p_{1}^{2} p_{2} \mathrm{e}^{-\frac{3 \alpha_{2} \lambda}{2}}+4 p_{1} p_{2}^{2} \mathrm{e}^{-\alpha_{2} \lambda}+p_{2}^{2}\left(2-p_{2}^{2}\right) \\
Q_{3} & =1-\mathrm{e}^{-\alpha_{3} \lambda}\left(1-Q_{2}\right)-4\left(1-\mathrm{e}^{-\frac{\alpha_{3} \lambda}{4}}\right) \mathrm{e}^{-\left(\alpha_{1}+\alpha_{2}+\frac{3 \alpha_{3}}{4}\right) \lambda} \\
\mu_{4} & =\left[1-\mathrm{e}^{-\alpha_{4} \lambda}\left(1-Q_{3}\right)\right] \mathrm{e}^{-\left(\gamma_{4}-1-3 \alpha_{1}-\alpha_{2}\right) \lambda} \\
\mu_{4}^{\mathrm{t}} & =\left(\alpha_{4} \lambda\right) \mathrm{e}^{-\gamma_{4} \lambda} .
\end{aligned}
$$

\section{Appendix B: Asymptotic expressions for measured count rates}

At high flux, the general method to evaluate integrals (5) and $(6)$ is to change variables to the local photon flux $\lambda=\Lambda g(x, y)$. I give here a few example calculations for an axisymmetric PSF $g(r)$.

For a King PSF, the asymptotic expression at high flux of the count rate $M_{1}^{\mathrm{t}}$ (Eq. 5) of clean (not piled-up) monopixels, can be written explicitly in the following way:

$g(r)=\frac{\beta / 2-1}{\pi r_{0}^{2}}\left(1+\left(r / r_{0}\right)^{2}\right)^{-\frac{\beta}{2}}$ 


$$
M_{1}^{\mathrm{t}} \simeq \frac{2}{\beta} \frac{\alpha_{1}}{\gamma_{1}} \pi r_{0}^{2}\left(\frac{\beta / 2-1}{\pi r_{0}^{2}}\right)^{\frac{2}{\beta}} \gamma_{1}^{\frac{2}{\beta}} \Gamma\left(1-\frac{2}{\beta}\right) \Lambda^{\frac{2}{\beta}}
$$

after changing variables to $v=\gamma_{1} \lambda$ and neglecting terms in $\mathrm{e}^{-\gamma_{1} \Lambda g(0)} . \Gamma$ denotes the Gamma function here.

The expectation value of the total count rate $M_{1}$ (Eq. 6) is somewhat more difficult to handle analytically but can also be written asymptotically in the following way:

$$
\begin{aligned}
& M_{1} \simeq \pi r_{0}^{2}\left(\frac{\frac{\beta}{2}-1}{\pi r_{0}^{2}}\right)^{\frac{2}{\beta}}\left[\gamma_{1}^{\frac{2}{\beta}}-\left(\gamma_{1}-\alpha_{1}\right)^{\frac{2}{\beta}}\right] \Gamma\left(1-\frac{2}{\beta}\right) \Lambda^{\frac{2}{\beta}} \\
& \frac{M_{1}^{\mathrm{t}}}{M_{1}} \simeq \frac{2}{\beta} \frac{\alpha_{1}}{\gamma_{1}}\left(1-\left(1-\frac{\alpha_{1}}{\gamma_{1}}\right)^{\frac{2}{\beta}}\right)^{-1}
\end{aligned}
$$

after splitting the integral in two, performing the same variable change (with $\gamma_{1}-\alpha_{1}$ instead of $\gamma_{1}$ for the first half), integrating by parts the $v^{-\frac{2}{\beta}-1}$ and the $\mathrm{e}^{-v}$ terms and noting that the two infinities at $v=0$ cancel out exactly.

This proves that for a PSF following a King profile the rates increase at high flux as $\Lambda^{2 / \beta}$ while the pile-up fraction tends to a (small) constant. The transition to this regime is when the incoming flux at the center gets higher than $\lambda_{0}$ of Eq. (4) and saturates.

For a Gaussian PSF (which has wings decreasing as fast as can be imagined for a reasonable PSF) the same kind of analytical exercise can be carried out:

$$
\begin{aligned}
& g(r)=\frac{1}{2 \pi r_{0}^{2}} \exp \left(-\frac{1}{2}\left(\frac{r}{r_{0}}\right)^{2}\right) \\
& M_{1}^{\mathrm{t}}=\frac{\alpha_{1}}{\gamma_{1}} 2 \pi r_{0}^{2}\left[1-\exp \left(-\frac{\gamma_{1} \Lambda}{2 \pi r_{0}^{2}}\right)\right] \\
& M_{1} \simeq 2 \pi r_{0}^{2} \ln \left(\frac{\gamma_{1}}{\gamma_{1}-\alpha_{1}}\right) \\
& \frac{M_{1}^{\mathrm{t}}}{M_{1}} \simeq \frac{\alpha_{1} / \gamma_{1}}{\left|\ln \left(1-\alpha_{1} / \gamma_{1}\right)\right|}
\end{aligned}
$$

after performing the same variable change as in (B2) (but with a different $g(r))$. (B5) is exact. In (B6) terms in $\mathrm{e}^{-\gamma_{1} \Lambda g(0)}$ were neglected. The main contribution to this integral comes from the difference between the two infinities at $v=0$, which can appear as

$\lim _{\epsilon \rightarrow 0} \int_{\left(\gamma_{1}-\alpha_{1}\right) \epsilon}^{\gamma_{1} \epsilon} \mathrm{e}^{-x} \frac{\mathrm{d} x}{x}=\ln \left(\frac{\gamma_{1}}{\gamma_{1}-\alpha_{1}}\right)$.

This shows that even for a Gaussian PSF the count rate never decreases as the flux increases, but tends to a constant instead. Because of the rapidly decreasing wings a Gaussian PSF leads to a higher pile-up fraction, reaching $6 \%$ for the worst case of only monopixel patterns.

\section{Appendix C: Example of application to real data}

\section{C.1. Presentation of the data}

The $X M M / E P I C$-MOS Flight Model 1 was calibrated at the Panter facility in January 1998 behind the $X M M$ mirrors (Sembay 1998), giving data with the spatial distribution of a point source in flight. Several runs were performed with the aim of calibrating pile-up. I have used the data taken with the Fe $\mathrm{L}$ source at varying beam intensity (runs 01343 to 01346), and the night-long run at low intensity (01351), in full-frame mode. The centering was the same in all these runs.

The data was cleaned as thoroughly as possible of bad pixels and background (using energy selection). The observed pattern distribution (outside the core, in order to be free of pile-up contamination) was [0.8834, 0.1040, $0.0061,0.0065]$. Patterns more complicated than quadripixels were negligible. The idea is not here to analyse in any detail the data itself, but simply to illustrate the relevance of the model to real data.

\section{C.2. Construction of the Point Spread Function}

This was not so easy for three reasons. Firstly there were clear deviations in the data from the ideal King profile. Secondly even the low intensity run could not be considered totally free of pile-up. Thirdly the number of counts in the wings of the low intensity run was inadequate to model the high intensity data. The problems were tackled in the following way:

- There were clear deviations in the data both from a simple King profile (Sembay 1998) and from the hypothesis of axial symmetry. Rather than try and invent a complicated analytical model, I decided to use the 2-D data directly.

- After normalizing the data of run 01351 to units of cts/pixel/frame, the total count rate in the low intensity run was $1.6 \mathrm{cts} /$ frame. The local count rate in the central pixel was larger than $0.01 \mathrm{cts} / \mathrm{pixel} /$ frame. The flux loss due to pile-up was therefore larger than $10 \%$ in the core, and had to be accounted for. The idea was to solve Eq. (2) for $\lambda$ at all pixels separately. For count rates lower than 0.01 (everywhere except the core), the correction was small and a second order development was used instead.

However this direct application was not very satisfactory because it amplified the statistical fluctuations in the data (about $10 \%$ in the core). Since $\mu_{1}$ in (2) should be the expectation value (not the measured value), the pixels above average were over-corrected and those below average under-corrected. The next idea was to smooth the data with a boxcar average of $3 \times 3$ pixels, prior to solving (2).

This was not acceptable either, because it resulted in smoothing the PSF itself with the same boxcar average, leading to $10 \%$ or so errors. The final solution (actually very close to solving the problem with large pixels, as presented in Sect. 4.4), was to proceed in two steps. In a first step, Eq. (2) was solved on the smoothed data, resulting in a smoothed PSF $\bar{\lambda}$. In a 


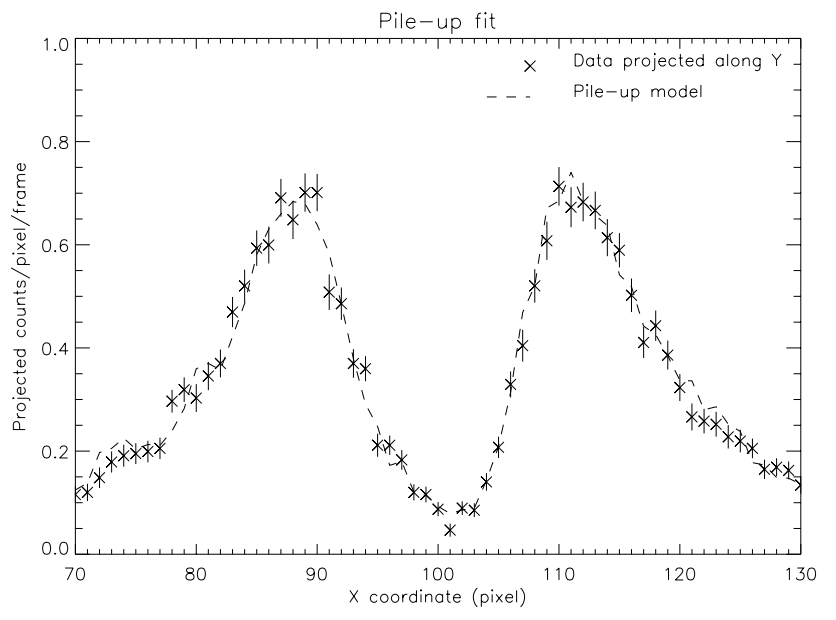

Fig. C1. Comparison between data obtained at Panter with the $X M M / E P I C-M O S$ in January 1998 at high intensity (run 01346) and the pile-up model according to (9). The data (crosses with error bars) and the model (shown as a dashed line) were limited to a band 21 pixels wide along $Y$ (centered on the source), and projected onto the $X$ axis. The source was centered at 100 . The total incoming flux was estimated to be 359 photons/frame (the measured count rate was 90 cts/frame). The PSF used in the model was derived as explained in C.2

second step, (2) was solved after replacing $\lambda$ by $\bar{\lambda}$ in the suppression term, resulting in

$\lambda=\frac{\ln \left(1+d \mathrm{e}^{\gamma_{1} \bar{\lambda}}\right)}{\alpha_{1}}$

where $d$ is the raw data. This allows to keep the original resolution while not over-correcting for pile-up. Of course the statistical fluctuations are still there, but there is no way to get rid of them without a model for the PSF.

- With the statistics at hand, the resulting PSF was very noisy. In particular the run at highest intensity (01346) had about 10 times more cts/pixel in the wings than the long low intensity run. In order to have the best PSF possible, I used data from run 01346 in the wings (distance larger than 30 pixels) where pile-up was not too large. This data was corrected for pile-up in the way described above, and smoothed with a boxcar average of variable width (from 3 pixels at distance 30 pixels to 17 at distance 100 and above). It was normalized to have the same integral in the wings as the PSF derived from the low intensity run. At distance between 10 and 30 pixels, the PSF (from run 01351) was also smoothed with a boxcar average of width 3 pixels to reduce its fluctuations (no true feature is expected at that distance at the pixel scale).

\section{C.3. Model comparison with piled-up data}

The comparison was performed for monopixels only. The events from runs 01343 to 01346 were projected onto an image and divided by the number of frames. The spatial domain was limited to a window of $201 \times 201$ pixels centered on the maximum. For each run Eq. (6) was solved for the incoming flux $\Lambda$ in the window, using the PSF constructed as described in C.2. Then the radial profiles, and profiles projected onto the $X$ and $Y$ axes, were compared with those expected from the pile-up model applied locally.

The comparison was fully satisfactory. It indicated that the fit was significantly better when one used Eqs. (9) than (2), and Fig. C1, illustrating the comparison, was obtained using (9). It is not clear, however, whether the improvement is due to the fact that even for $X M M / E P I C$ $M O S$ the pixels are not small enough to allow using (2) with a high precision, or that the statistical fluctuations in the model PSF lead to the wrong pile-up corrections (as described in C.2). Data with higher statistical significance in the PSF core would be required to decide.

\section{References}

EPIC MOS team, X-ray Astronomy Group, Leicester University and Service d'Astrophysique, CEA Saclay, 1994, "EPIC MOS EOBB final report"

Erd C., Much R., Lumb D., 1996, XMM-PS-GM-01, Issue 2, "Description of the basic modes of the experiments on board the XMM observatory"

Ferrando P., Lortholary M., Pigot C., 1996, EPIC document $\mathrm{SAP} / \mathrm{PF} / 04-06-96$, "Pile-up rates and pile-up recovery for the EPIC-MOS CCDs: a Monte-Carlo study"

Lumb D.H., Berthiaume G.D., Burrows D.N., et al., 1991, Exptl. Astron. 2.3, 179

Sembay S., 1998, EPIC Consortium Meeting, Garching 23-25/06/98, "MOS Panter calibration overview"

Villa G.E., Abbey A.F., Arnaud M., et al., 1996, Proc. SPIE 2808,402 